\title{
Docosahexaenoic Acid Monoglyceride Increases Carboplatin Activity in Lung Cancer Models by Targeting EGFR
}

\author{
CAROLINE MORIN ${ }^{1}$ and SAMUEL FORTIN ${ }^{1,2}$ \\ ${ }^{1}$ SCF Pharma, Ste-Luce, QC, Canada; \\ ${ }^{2}$ Department of Biology, Université du Québec à Rimouski, Rimouski, QC, Canada
}

\begin{abstract}
Background/Aim: Omega 3 polyunsaturated fatty acids (PUFAs) have been shown to inhibit the induction and progression of many tumor types. The aim of this study was to determine the anticancer effect of docosahexaenoic acid monoglyceride (MAG-DHA) alone and in combination with the chemotherapeutic agent carboplatin (CBT) on lung cancer models. Materials and Methods: Adenocarcinoma cell lines A549 and H1299 were used to evaluate the effect of combined MAG-DHA and CBT treatments both in vitro and in vivo in xenograft models. Results: MAG-DHA+CBT treatment decreased cell proliferation and invasion abilities of A549 and H1299 cells. Furthermore, MAG-DHA+CBT treatment resulted in a decreased activation of epithelial growth factor receptor (EGFR) and its downstream extracellular signal-regulated kinase (ERK) in cell lysates. In A549 and H1299 xenograft mouse models, MAG$D H A+C B T$ treatment reduced tumor growth. Conclusion: Combined MAG-DHA and CBT treatment inhibited tumor growth by suppressing EGFR and ERK signaling pathways in lung carcinoma cells.
\end{abstract}

Lung cancer is the leading cause of cancer mortality in North America with non-small cell lung cancer (NSCLC) accounting for over $75 \%$ of lung cancer patients (1). Studies suggest that omega-3 polyunsaturated fatty acids (PUFAs), such as eicosapentaenoic acid (EPA) and docosahexaenoic acid (DHA) display chemopreventive and therapeutic potential against lung cancer (2). Dietary intake of PUFAs is associated with a lower risk of developing cancer $(3,4)$, and inhibits both the growth and metastasis of animal tumors and xenografts (2-4).

This article is freely accessible online.

Correspondence to: Samuel Fortin, SCF Pharma, 235 route du Fleuve Ouest, G0K 1P0, Ste-Luce, QC, Canada. Tel: +1 5143179347, e-mail: sfortin@ scfpharma.com

Key Words: DHA, carboplatin, lung carcinoma, EGFR.
However, the anticancer properties and mechanism of action of omega-3 PUFAs in lung cancer remain unclear. Some studies link the tumor-suppressive activity of omega-3 PUFAs with the mitogen-activated protein kinase pathway and either the activation or inactivation of its components with the induction of apoptosis (5) and growth arrest (6). Our previous studies have demonstrated that well-absorbed omega-3 PUFAs, docosapentaenoic acid monoglyceride (MAG-DPA) and MAGDHA are able to exert anti-proliferative and pro-apoptotic effects on colorectal and lung cancer cells through the downregulation of NFKB and up-regulation of PTEN $(7,8)$.

Several experimental studies using a variety of chemotherapeutic agents and tumor types have reported a greater efficacy of chemotherapy with the addition of omega3 PUFA $(9,10)$. EPA and DHA have been shown to increase tumor sensitivity to irinotecan therapy and protect non-target tissues (11). Murphy et al. have shown that the administration of an EPA+DHA supplement to NSCLC patients undergoing platinum-based chemotherapy caused a two-fold increase in therapy response rate and clinical benefit when compared with patients undergoing the same treatment without additional supplementation (12). Improved chemotherapy outcomes have been observed in breast cancer patients undergoing anthracycline-based chemotherapy when a daily DHA supplement was highly incorporated into circulating phospholipids (13). However, the mechanism explaining the increased sensitivity to chemotherapy induced by omega-3 PUFAs remains unclear.

Overexpression or mutations in intracellular epidermal growth factor receptor (EGFR) have been reported in $43-89 \%$ of cases and have been implicated in the pathogenesis of many human malignancies, including NSCLC (14). These mutations can result in constitutive activation of signal transduction pathways, leading to cell proliferation or anti-apoptosis, regardless the presence of extracellular ligand (15). Some studies have shown that EGFR expression in NSCLC is associated with reduced survival, frequent lymph node metastasis, and poor chemosensitivity (16-18). Upon binding of a specific ligand (epidermal growth factor, EGF), the 
normally functioning EGFR undergoes conformational change and phosphorylation of the intracellular domain occurs, leading to downstream signal transduction by various pathways (14). These include the Ras/Raf/MAPK (mitogen-activated protein kinase) pathway, the PI3K (phosphatidylinositol-3-kinase)/Akt pathway and the JAK (Janus kinase)/STAT (signal transducer and activator of transcription pathway $(14,15)$. Depending on the pathway that is activated, the end result is cell proliferation or cell maintenance by inhibition of apoptosis (14). Hence, it would be of potential clinical interest to delineate a specific agent that could target EGFR and oppose its effect on tumor cells.

Despite the emergence of new targeted agents and the use of various therapeutic combinations, none of the treatment options available is curative in patients with advanced NSCLC (1). The aim of the present study was to evaluate the anti-tumorigenic effects of MAG-DHA alone and in combination with carboplatin (CBT) chemotherapy. Hence, we evaluated the in vitro effect of MAG-DHA on tumor cell proliferation related to EGFR pathway. Two human lung cancer cell lines, A549 and H1299 have been used. Moreover, in vivo mouse models of A549 and H1299 xenografts were also used to evaluate whether per os MAGDHA and intraperitoneal (ip) CBT treatments exert antiproliferative effects through EGFR pathway inhibition.

\section{Materials and Methods}

MAG-DHA. MAG-DHA (Solutex, Madrid, Spain) is a fish oil concentrate in monoglyceride form containing $621 \mathrm{mg} / \mathrm{g}$ DHA, 107 $\mathrm{mg} / \mathrm{g}$ DPA and $27 \mathrm{mg} / \mathrm{g}$ EPA $(19,20)$.

Cell culture. Human normal epithelial BEAS-2B cells as well as A549 and H1299 non-small cell lung carcinoma cell lines were obtained from the American Type Culture Collection (ATCC, Manassas, VA, USA). A549 and H1299 cells were maintained in RPMI 1640 (Wisent, St-Bruno, QC, Canada) containing 10\% FBS (Wisent, St-Bruno, QC, Canada) and $10 \mathrm{units} / \mathrm{ml}$ penicillin, $100 \mu \mathrm{g} / \mathrm{ml}$ streptomycin (Wisent, St-Bruno, QC, Canada). BEAS-2B cells were maintained in BEBM medium (Lonza, Inc, Allendale, NJ, USA) in culture flasks pre-coated with a mixture of $0.01 \mathrm{mg} / \mathrm{ml}$ fibronectin (Wisent, St-Bruno, QC, Canada), $0.03 \mathrm{mg} / \mathrm{ml}$ bovine collagen type I (Wisent, St-Bruno, QC, Canada) and $0.01 \mathrm{mg} / \mathrm{ml}$ serum albumin (Wisent, St-Bruno, QC, Canada) dissolved in BEBM medium. Cells were grown in a $5 \% \mathrm{CO}_{2}$ incubator at $37^{\circ} \mathrm{C}$ and used between passages 3 to 6 for all conditions and assays tested in this study. Cells were untreated or treated with MAG-DHA and CBT (Sigma-Aldrich, Oakville, ON, Canada) at the indicated concentrations.

Cell proliferation and invasion analyses. Cell proliferation analyses were performed using the BrdU cell proliferation assay kit (New England BioLabs, Pickering, ON, Canada). Briefly, A549 or H1299 cell suspensions containing 2,500 cells/ml in RPMI medium were plated in 96-well plate and incubate with MAG-DHA, CBT, or MAG-DHA+CBT for $48 \mathrm{~h}$ at $37^{\circ} \mathrm{C}$ in $5 \% \mathrm{CO}_{2}$. BrdU solution was added in the last $24 \mathrm{~h}$ of incubation. Typical ELISA assay were performed using manufacturer's instructions. Relative Light Units (RLU) signals were measured at $425 \mathrm{nM}$ within 1-10 min following addition of the substrate, using a plate-reader (Perkin Elmer, Woodbridge, ON, Canada).

Cell invasion analyses were performed using the 24-well Cytoselect Cell Invasion assay in accordance with the manufacturer's instructions (Cell Biolabs Inc, San Diego, CA, USA). Briefly, A549 or H1299 cell suspensions containing $0.5 \times 10^{6}$ cells $/ \mathrm{ml}$ in RPMI medium were added on the cell culture inserts. Cells were incubated for $48 \mathrm{~h}$ at $37^{\circ} \mathrm{C}$ in $5 \% \mathrm{CO}_{2}$ in the absence (control) or presence of MAG-DHA, CBT, or MAG-DHA+CBT. Non-invasive cells were removed and invasive cells were stained and counted using a light microscope. For colorimetric analysis, each cell culture insert was transferred in a new 24 well-plate and extraction solution was added. Finally, $100 \mu \mathrm{l}$ from each well were transferred to a 96-well microtiter plate and the OD $560 \mathrm{~nm}$ was measured using plate reader (Perkin Elmer, Woodbridge, ON, Canada).

Western blot analysis. Western blots using specific antibodies against EGFR, ERK1/2, $\beta$-actin and phosphorylated forms of EGFR and ERK1/2 (all from New England BioLabs, Pickering, ON, Canada) were performed on cell lysate fractions as previously described (8). Antibodies were used at a concentration of $1 \mu \mathrm{g} / \mathrm{ml}$ for overnight incubation at $4^{\circ} \mathrm{C}$. Immunostaining of the blots were digitized and analyzed with Lab-Image software 2.7 (Kapelan Bio-Imaging, Leipzig, Germany). Blots were stripped using reblot plus strong antibody stripping solution, (Millipore, Etobicoke, ON, Canada) and reprobed with total antibody against EGFR or ERK1/2 and $\beta$-actin.

In vivo tumor xenograft experiments. Female nu/nu nude mice were obtained from Charles River Laboratories (Montreal, QC, Canada). All studies involving mice were approved by the institutional Animal Care Committee (Protocol: \# 237-10). Human A549 and H1299 xenografts were established in 4-week-old nude mice. Mice were subcutaneously inoculated with $0.2 \mathrm{ml}$ of $1 \times 10^{6}$ cells in RPMI on the right flank. After formation of $50 \mathrm{~mm}^{3}$ or $100 \mathrm{~mm}^{3}$ tumors, mice were randomly assigned into 4 groups, control (animals received $618 \mathrm{mg} / \mathrm{kg}$ PBS per os), MAG-DHA-treated, MAG$\mathrm{DHA}+\mathrm{CBT}$ and CBT (n=6 per group). MAG-DHA was administered per os $(618 \mathrm{mg} / \mathrm{kg})$ daily following tumor formation. CBT was administered ip $(15 \mathrm{mg} / \mathrm{kg})$ every 7 days following tumor formation. To measure the effect of MAG-DHA and CBT treatments on progression of tumor growth, tumor dimensions (length and width) were measured by a caliper and tumor volume was calculated by the formula: (length $\times$ width $\left.^{2}\right) / 2$.

Data analysis and statistics. Results are expressed as mean \pm S.E.M., with $\mathrm{n}$ indicating the number of experiments. Statistical analyses were performed using Sigma Plot 11 and SPSS 14.0 (SPSS-Science, Chicago, IL) via one-way ANOVA followed by Dunnett's post-hoc test. Differences were considered statistically significant when $p<0.05$.

\section{Results}

Effect of MAG-DHA and CBT treatments on cell proliferation. Experiments were performed to assess the effect of MAG-DHA treatments on cell proliferation in human normal bronchial epithelial cell BEAS-2B and lung adenocarcinoma A549 and H1299 cells. The cells were treated with increasing concentrations of MAG-DHA $(0.01$, 
$0.03,0.1,0.3,1,3,10,30$ and $100 \mu \mathrm{M})$ for $48 \mathrm{~h}$ and cell proliferation rate was assessed by BrdU incorporation assay. Cumulative concentration response curves revealed that MAG-DHA decreased the proliferation rate of BEAS-2B, A549, and $\mathrm{H} 1299$ cells, with $\mathrm{IC}_{50}$ values of $42.9 \pm 0.02 \mu \mathrm{M}$, $1.85 \pm 0.03 \mu \mathrm{M}$ and $3.90 \pm 0.04 \mu \mathrm{M}$, respectively (Figure $1 \mathrm{~A}$ ). Combined MAG-DHA and CBT treatment was evaluated on A549 and H1299 cell proliferation using the BrdU assay. Results revealed that combined MAG-DHA and $0.1 \mu \mathrm{M}$ CBT treatment have an additive effect on the inhibition of cell proliferation inhibition in A549 cells $(p<0.05)$ (Figure 1B). Moreover, Figure 1B shows a higher inhibitive effect of increased CBT concentration $(0.3 \mu \mathrm{M})$ plus MAG-DHA on A549 cells. Similar experiment was performed on H1299 cells and quantitative analyses revealed that combined $0.3 \mu \mathrm{M}$ CBT and $1 \mu \mathrm{M}$ MAG-DHA treatment results in a greater inhibitory effect on cell proliferation $(p<0.05)$ (Figure 1C).

Effect of MAG-DHA and CBT on cell invasion. The effects of MAG-DHA and CBT treatment on the invasive abilities of A549 and H1299 lung adenocarcinoma cells were evaluated using an in vitro cell invasion assay. Microscopic images demonstrated that $1 \mu \mathrm{M}$ MAG-DHA treatment on A549 and H1 299 cells for 48h reduced their invasiveness (Figure 2A and 2C). Data analysis revealed $60 \pm 3 \%$ and $51 \pm 4 \%$ reduction of invasive A549 and H1299 cells, respectively. The effect of CBT $(0.1 \mu \mathrm{M})$ in combination with MAG-DHA on invasion properties of lung adenocarcinoma cells were also evaluated (Figure 2B). Data revealed that combined treatment results in additive effect on reduction of A549 cells invasion when compared to the effect induced by CBT or MAG-DHA alone, with a $p$-value $<0.05$. Similar results were observed following MAG-DHA+CBT $(0.1 \mu \mathrm{M})$ treatment on $\mathrm{H} 1299$ cells, and we quantified significant $47 \%$ reduction of invasive cells $(p<0.05)$.

Effect of MAG-DHA and CBT on EGFR and ERK activation. Western blot analyses were performed to evaluate the activation of EGFR and ERK on both cell lineages in control conditions and after treatment with $0.1 \mu \mathrm{M}$ CBT alone, $1 \mu \mathrm{M}$ MAG-DHA alone, and combined $0.1 \mu \mathrm{M}$ CBT and $1 \mu \mathrm{M}$ MAG-DHA. Western blots were performed on A549 and H1299 cells lysates using antibodies against $\beta$-actin (loading control) as well as against total and phosphorylated forms of EGFR (Figure 3A). Data analyses revealed that $1 \mu \mathrm{M}$ MAG-DHA treatment significantly reduced phosphorylation (P-EGFR) and total expression level of EGFR $(p<0.05)$ (Figure 3B) in A549 and H1299 cell lines. CBT treatment alone did not affect the phosphorylation and expression level of EGFR when compared to the level of control (untreated) A549 and H1299 cells. However, results from both cell lines revealed that combined MAG-DHA and CBT treatment significantly decreased the phosphorylation and total expression levels of EGFR comparatively to controls (Figure 3A-B). Moreover, a significant decrease in EGFR phosphorylation and expression levels was observed between MAG-DHA-treated and combined MAG-DHA+CBT-treated A549 cells (Figure 3B). Western blot and quantitative immunoblot analyses were also performed on A549 and H1299 cell lysates derived from control, CBT, MAGDHA and MAG-DHA+CBT-treated cells using antibodies against total and phosphorylated forms of ERK1/2. Results revealed that combined MAG-DHA and CBT treatment decreased ERK phosphorylation levels in treated cells compared to untreated controls (Figure 3C-D). Significant reductions were observed between MAG-DHA plus CBT-treated and MAGDHA-treated groups of A549 and H1299 cells (43\% and 81\%, respectively, $p<0.05$ ), following comparative analysis of $\mathrm{P}$ ERK/ERK ratios (Figure 2D). The expression level of ERK1/2 was fairly constant between preparations for both adenocarcinoma cell lines.

Effect of MAG-DHA and CBT treatments on A549-induced tumor formation. Xenograft mice model was used to determine the effect of MAG-DHA on the ability of A549 cells to form tumors following their subcutaneous injection. Following tumor formation of $50 \mathrm{~mm}^{3}$ mice were randomly divided in 4 groups, namely, control, CBT, MAG-DHA and MAG-DHA+CBT-treated mice. As shown in the diagram illustrated on Figure 4A, mice were treated with MAG-DHA per os $(618 \mathrm{mg} / \mathrm{kg})$ daily or/and CBT ip $(15 \mathrm{mg} / \mathrm{kg})$ every 7 days following tumor formation of $50 \mathrm{~mm}^{3}$. Mice of MAGDHA group received 37 doses of MAG-DHA (day 57) and treatment was stopped on days 58 to 102 . Data analysis revealed that MAG-DHA treatment significantly inhibited the growth of A549 xenografts compared to control animals (Figure 4B): Moreover, no tumor was detected on 2 mice of MAG-DHA-treated group on day 102, and the mean tumors volume of 4 other mice was $12 \pm 2 \mathrm{~mm}^{3}$ (Figure 4B). In MAG-DHA+CBT group, mice received 6 injections of CBT and 37 doses of MAG-DHA and all treatments were stopped on day 58 to 102. Results demonstrated that tumor growth reduction in MAG-DHA+CBT-treated mice was similar to MAG-DHA-treated mice alone. However, at the end of the experiment (day 102), no tumor was detected in 5 mice (Figure 4B). No significant difference was observed between control and CBT-treated mice on the ability of A549 cells to form tumors. Representative macroscopic images of tumor size illustrated in Figure 4C revealed the potent effect of MAG-DHA and MAG-DHA+CBT treatments when compared to control and CBT-treated mice. No significant difference in mice weight was observed between groups.

Effect of combined MAG-DHA and CBT treatment on $H 1299$ xenografts. Experiments were designed to determine the tumor-inhibitory activity of MAG-DHA and CBT in a H1299 xenograft mice model. Following tumor formation of $100 \mathrm{~mm}^{3}$, corresponding to 20 days after injection of 
A

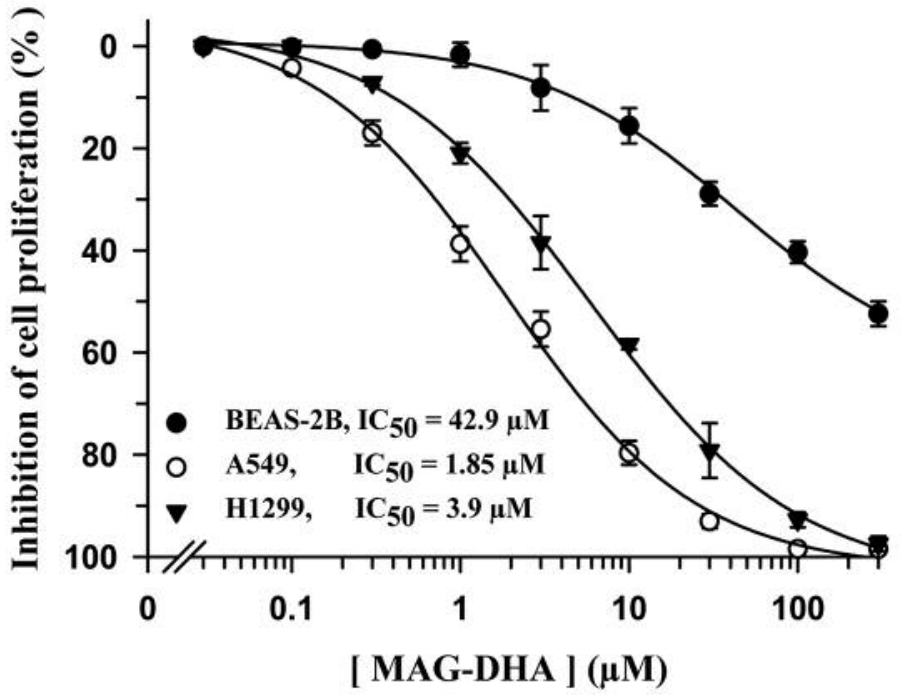

B

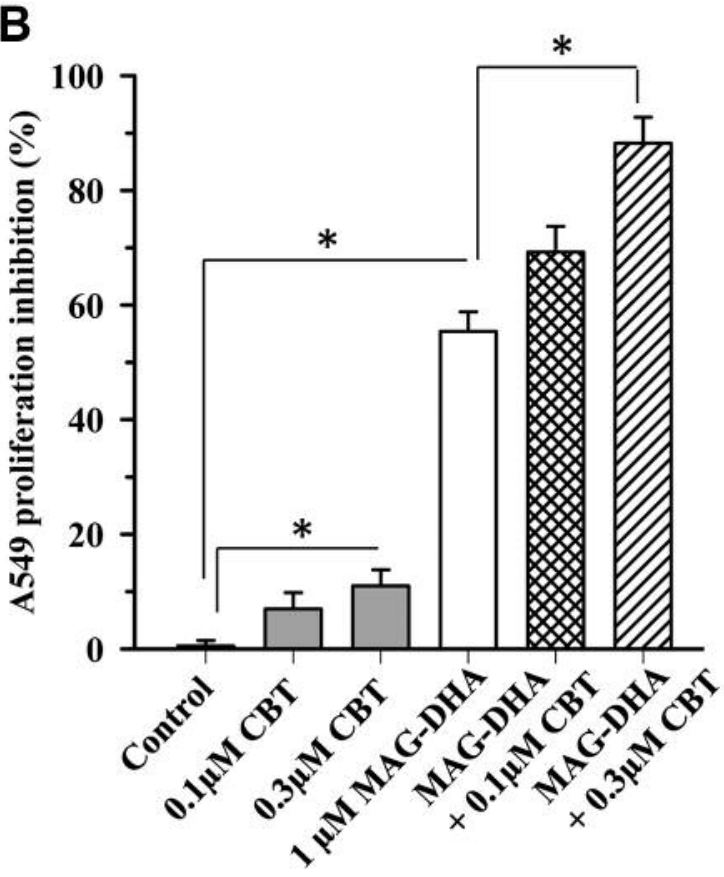

C

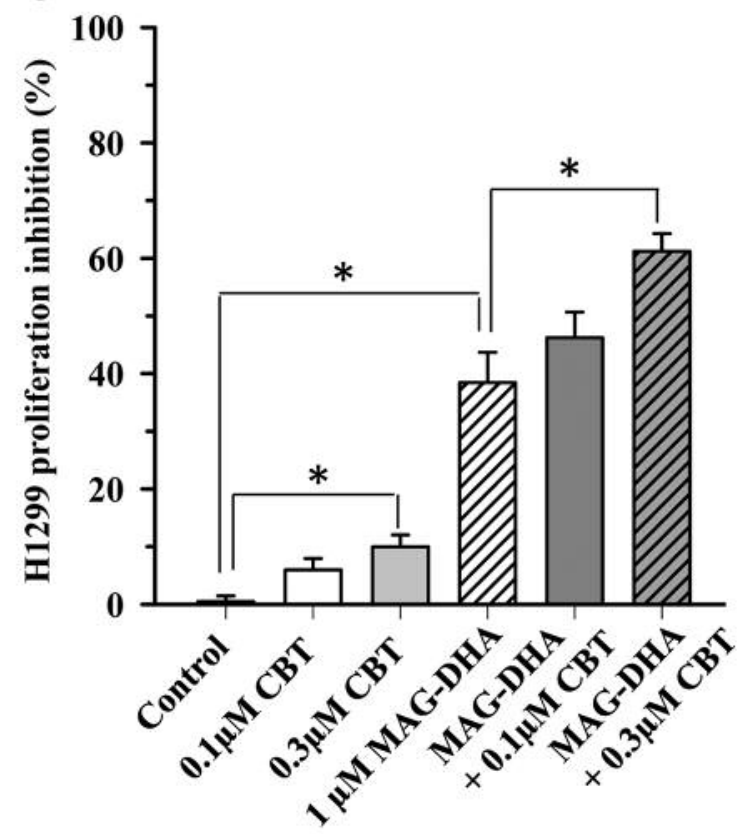

Figure 1. Effect of MAG-DHA and CBT treatments on proliferation of A549 and H1299 cells. A: Cumulative concentration response curves display the inhibitory effect of MAG-DHA on normal epithelial cells BEASE2B and in tumoral lung epithelial cells A549 and H1299, evaluated using the BrdU proliferation assay ( $n=8$ for each experimental condition). B: Bar histogram displays the mean percent inhibition of cell proliferation of A549 control cells or treated with CBT $(0.1$ and $0.3 \mu M), M A G-D H A(1 \mu M$,) and combined MAG-DHA (1 $\mu M)$ plus CBT $(0.1 \mu M)(n=8)$. C: Bar histogram displays the mean percent inhibition of cell proliferation of $H 1299$ control cells or treated with CBT (0.1 and 0.3 $\mu M)$, MAG-DHA $(1 \mu M)$, and combined MAG-DHA $(1 \mu M)$ plus CBT $(0.1 \mu M)(n=6)$. Asterisc $\left(^{*}\right)$ indicates statistical significance p<0.05. MAG-DHA, Docosahexaenoic acid monoglyceride; CBT, carboplatin.

$1 \times 10^{6} \mathrm{H} 1299$ cells in the right flank of nude mice, animals were randomly divided in three groups, namely control, MAG-DHA and MAG-DHA+CBT. MAG-DHA was per os administrated daily $(618 \mathrm{mg} / \mathrm{kg})$ at day 20 to 53 and experiment was terminated on day 54 (Figure 5A). As shown in Figure 5B, MAG-DHA treatment significantly inhibited the growth of H1299 xenografts compared to control mice $(p<0.05)$. In MAG-DHA+CBT group, mice 
A

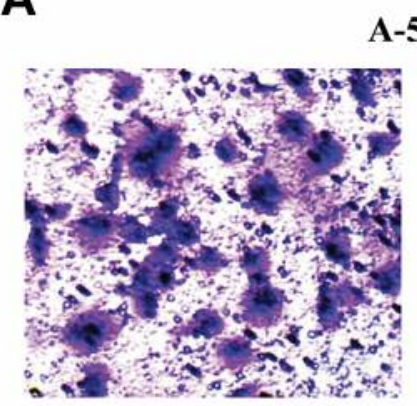

Control
A-549

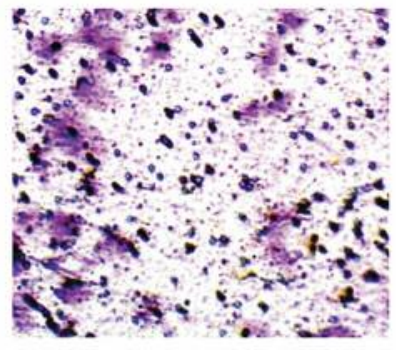

MAG-DHA

C

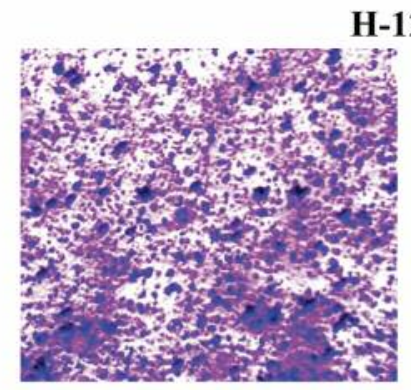

Control
H-1299

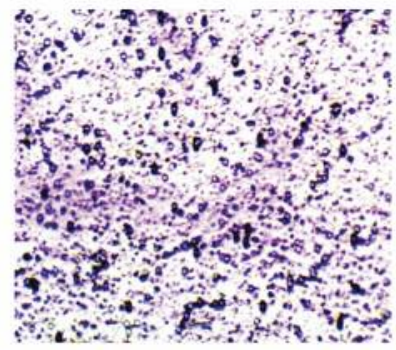

MAG-DHA

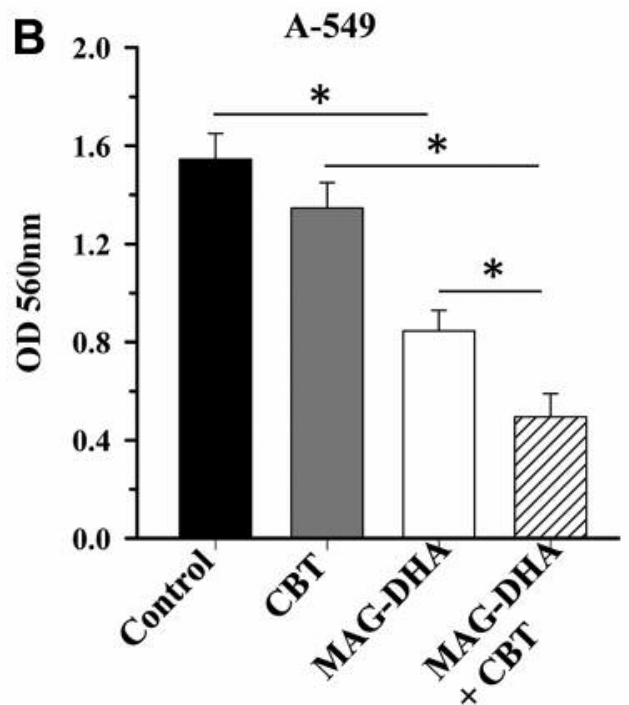

D $\quad$ H-1299

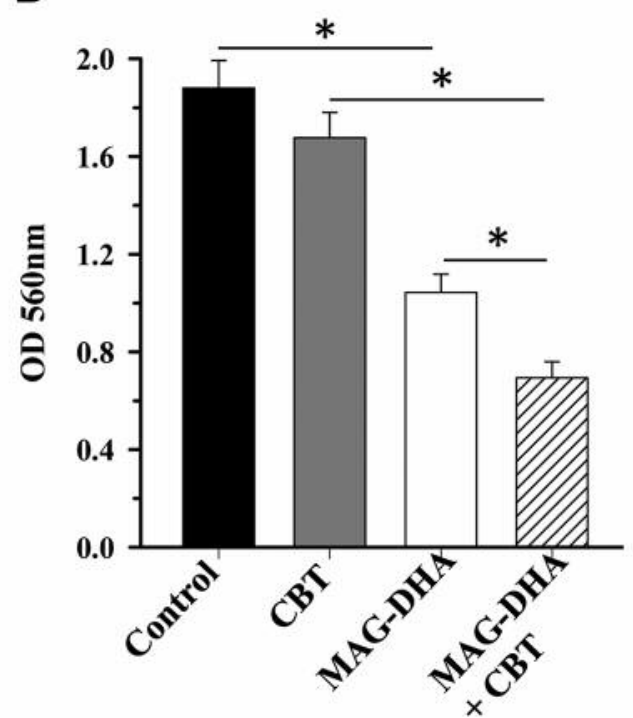

Figure 2. Effect of MAG-DHA treatment on the invasive abilities of A549 and H1299 cells. A: Representative images showing invasive cells (blue) in control and $1 \mu M$ MAG-DHA-treated A549 cells following cell invasion assay. B: Bar histogram displaying quantitative analysis of invasive cells quantified by spectrometry (OD 560nm) in control A549 cells, and after treatment with $0.1 \mu M C B T, 1 \mu M$ MAG-DHA, or combined $0.1 \mu M$ CBT plus $1 \mu \mathrm{M}$ MAG-DHA ( $n=6$ for each experimental condition). C: Representative images showing invasive cells (blue) in control and $1 \mu M \mathrm{MAG}-$ DHA-treated H1299 cells following in vitro assay. D: Bar histogram displaying quantitative analysis of invasive cells in control and treated H1299 cells with $0.1 \mu M C B T, 1 \mu M M A G-D H A$, or $0.1 \mu M C B T+1 \mu M M A G-D H A(n=6$ for each experimental condition). Asterisc (*) indicates statistical significance $p<0.05$, MAG-DHA, Docosahexaenoic acid monoglyceride; CBT, carboplatin.

received 5 injections of CBT ip $(15 \mathrm{mg} / \mathrm{kg})$ every 7 days and daily doses $(618 \mathrm{mg} / \mathrm{kg})$ of MAG-DHA following tumor formation of $100 \mathrm{~mm}^{3}$. Data clearly demonstrated that tumor growth inhibition was similar in MAGDHA+CBT-treated mice and MAG-DHA-treated mice alone.

\section{Discussion}

In the present study, combined MAG-DHA and chemotherapy agent CBT treatments were found to reduce cell proliferation and invasion of lung adenocarcinoma cells in vitro and in vivo in mouse xenograft models. These effects 


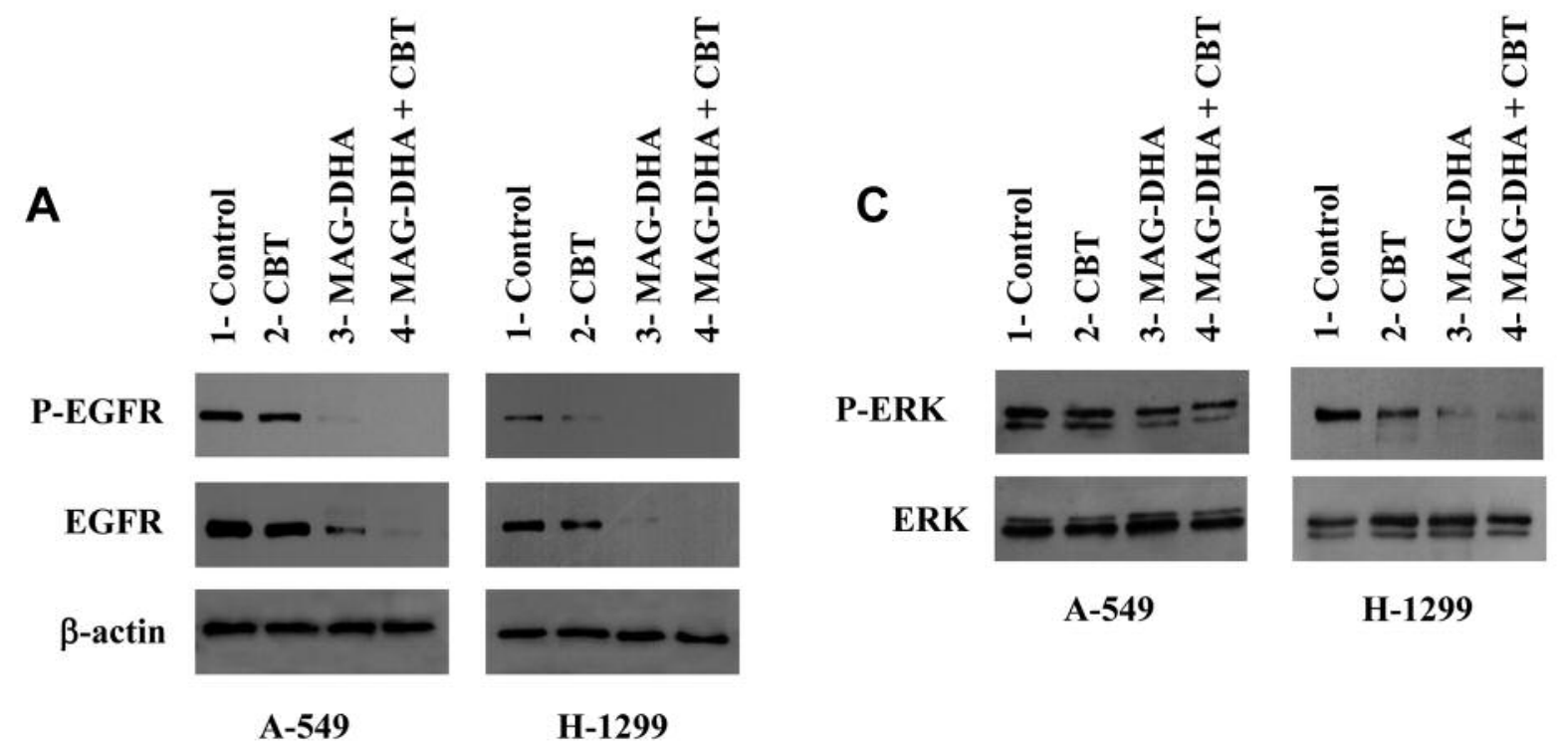

B A-549

H-1299

D

A-549

H-1299
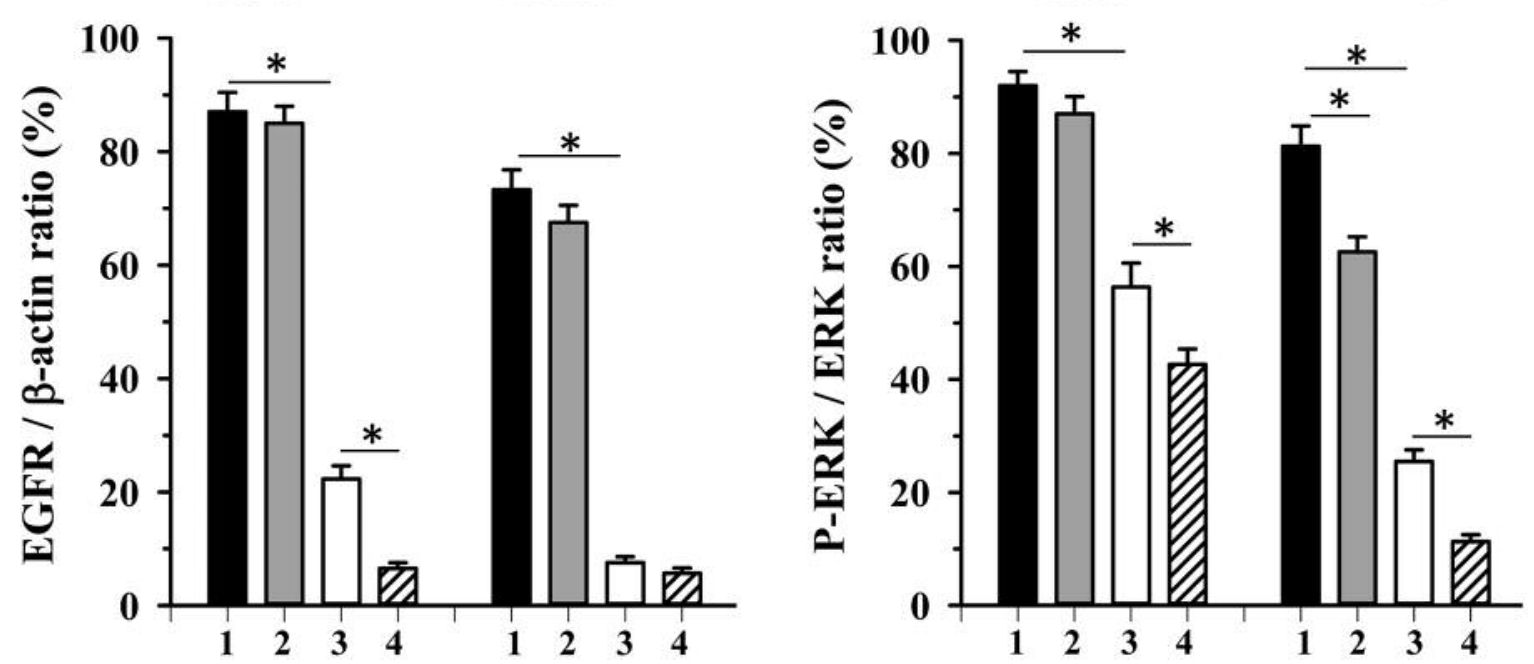

Figure 3. Effect of combined MAG-DHA and CBT treatments on EGFR and ERK phosphorylation and expression levels in A549 and H1299 cells lysates. A: Western blot analysis of A549 and H1299 cell lysates derived from control (1), CBT (2), MAG-DHA (3) and MAG-DPA+CBT (4)-treated cells using specific antibodies against the phosphorylated form of EGFR (P-EGFR) and total form of EGFR and $\beta$-actin protein detection. B: Quantitative analyses of EGFR density ratios. Staining densities of EGFR in A549 and $H 1299$ cell lysates were expressed as a function of $\beta$-actin signals. (n=6, $p<0.05)$. Each number 1-4 corresponds to a different experimental group. C: Western blot analysis of phosphorylated form of ERK1/2 (P-ERK) and total form of ERK1/2 protein detection in $A 549$ and H1299 cells lysates derived from control (1), CBT (2), MAG-DHA (3) and MAG-DHA+CBT (4)-treated cells. D: Quantitative analyses of P-ERK density ratios. Staining densities in A549 and H1299 cells lysates were expressed as a function of total ERK signals $(n=6)$. Each number $1-4$ corresponds to a different experimental group. Asterisc $(*)$ indicates statistical significance p<0.05. MAG-DHA, Docosahexaenoic acid monoglyceride; CBT, carboplatin; EGFR, epidermal growth factor receptor; ERK, extracellular signal-regulated kinase.

of MAG-DHA and CBT were correlated with a reduction in phosphorylation and expression levels of EGFR.

MAG-DHA and CBT reduced proliferation and invasiveness of lung cancer cells. Several in vitro and in vivo studies have shown that omega-3 PUFA supplementation prevent or reduce the growth of tumor cells $(2,3,5-8)$. Moreover, clinical studies have demonstrated significant protective effects of omega-3 fatty acids against lung cancer and high omega- 3 intake was associated with a lower risk of lung cancer $(3,4,21)$. However, 


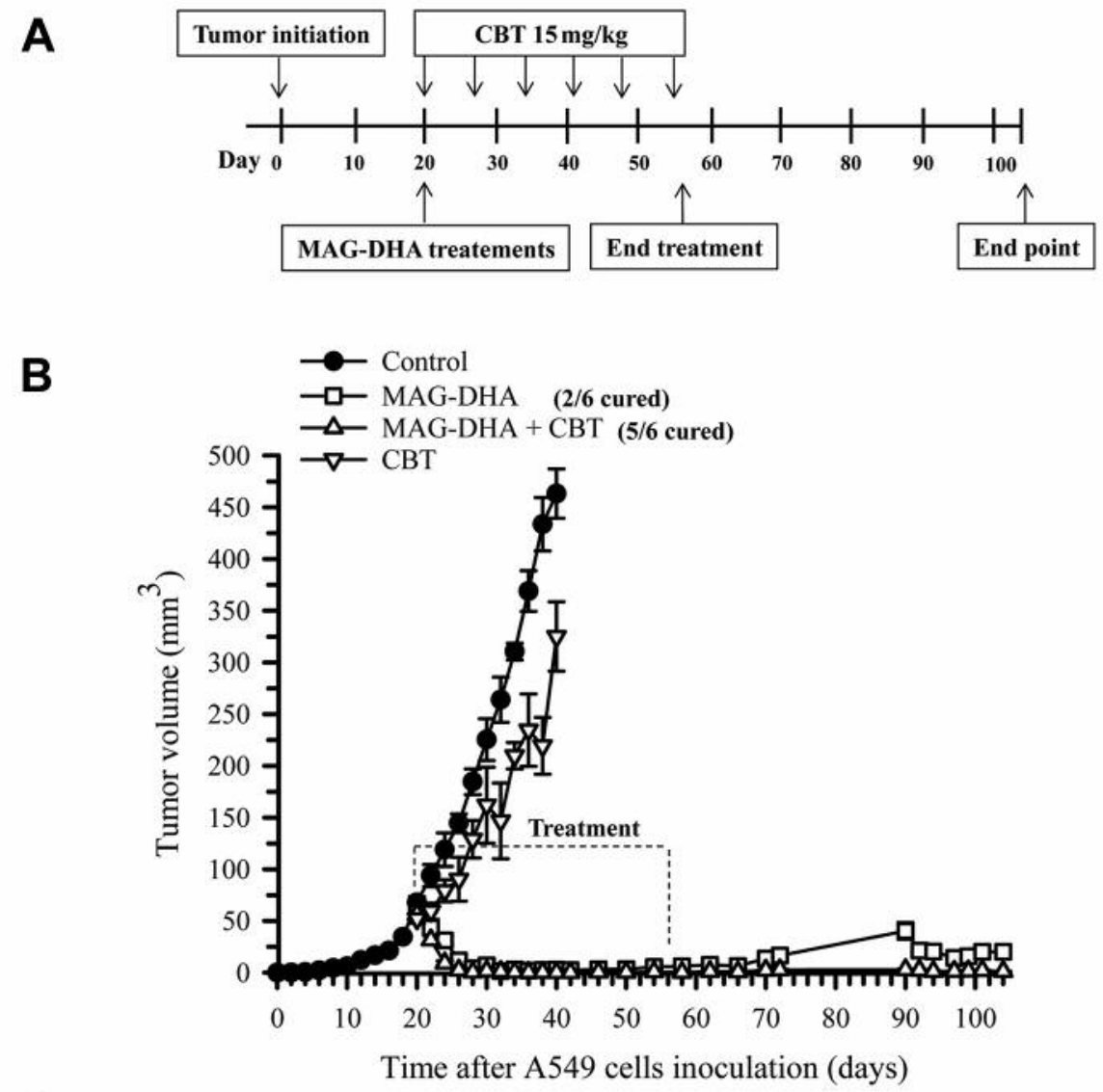

C

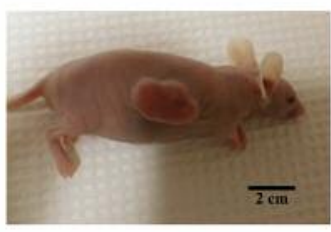

Control

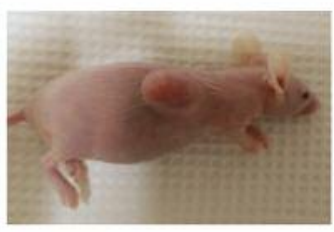

CBT

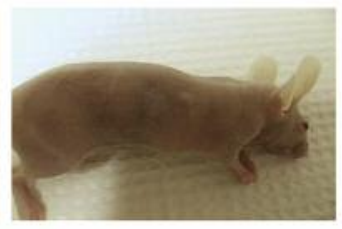

MAG-DHA

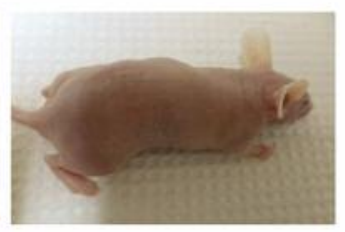

MAG-DHA + CBT

Figure 4. Effects of combined MAG-DHA and CBT treatment on tumor growth in the A549 xenograft nude mice model. A: Diagram showing the murine $A 549$ xenograft protocol and treatments as a function of time (day). MAG-DHA was administered per os (618 mg/kg) daily following tumor formation of $50 \mathrm{~mm}^{3}$ (day 20). CBT was administered i.p. $(15 \mathrm{mg} / \mathrm{kg})$ every 7 days following tumor formation of $50 \mathrm{~mm}^{3}$. Mice received 6 injections of CBT until the end of MAG-DHA treatment (day 57). For MAG-DHA and MAG-DHA+CBT groups no treatment was given between days 58 and 102. B: Tumor growth $\left(\mathrm{mm}^{3}\right)$ as a function of time (day) was measured after subcutaneous injection of $1 \times 10^{6} \mathrm{~A} 549 \mathrm{cells}$ in control, CBT $15 \mathrm{mg} / \mathrm{kg}$ ), $M A G-D H A(618 \mathrm{mg} / \mathrm{kg})$ and MAG-DHA+CBT-treated nude mice. The control and CBT mice were sacrificed following tumor formation of $500 \mathrm{~mm}^{3}$ on day 40, whereas the mice of MAG-DHA and MAG-DHA+CBT groups were sacrificed on day 103. Results represent the mean tumor volume $\pm S E M$ ( $n=6$ per group, $p<0.05$ ). C: Representative macroscopic images of tumor size derived from control, CBT, MAG-DHA and MAG-DHA+CBT-treated mice. MAG-DHA, Docosahexaenoic acid monoglyceride; CBT, carboplatin.

to date no study has demonstrated the impact of combined treatments with DHA monoglyceride (MAG-DHA) and chemotherapeutic agent $\mathrm{CBT}$ in in vitro and in vivo models related to lung cancer. Herein, we reported that a greater efficacy of CBT with the addition of MAG-DHA to reduce growth and proliferation of A549 and H1299 cells. In in vivo
A549 xenograft models, combined MAG-DHA and CBT administration were able to cure $80 \%$ of animals following 37 days of treatment. Clinical studies have shown that omega-3 PUFAs increased tumor sensitivity to a variety of chemotherapeutic agents and protect non-target tissues $(11,13)$ Moreover, a clinical trial has shown that supplementation with 

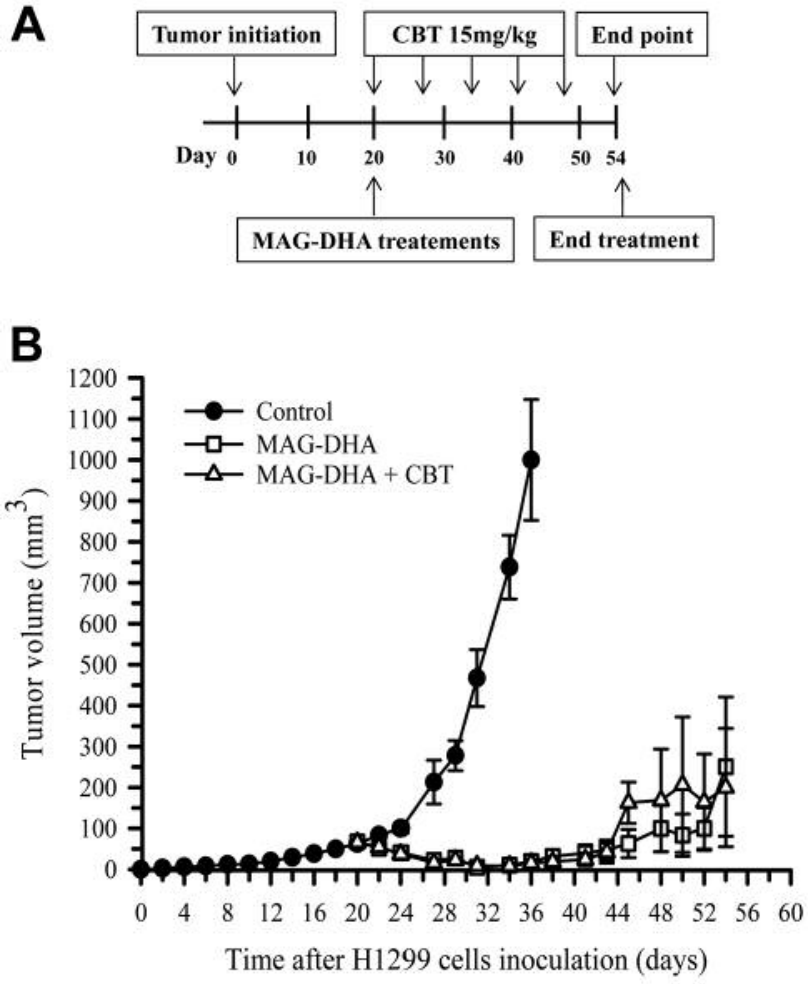

Figure 5. Effects of combined MAG-DHA and CBT treatment on tumor growth in the H1299 xenograft mice model. A: Diagram showing the H1299 xenograft protocol and treatments as a function of time (day). MAG-DHA was administered per os $(618 \mathrm{mg} / \mathrm{kg})$ daily following tumor formation of $50 \mathrm{~mm}^{3}$ (day 20). CBT was administered i.p. (15 mg/kg) every 7 days following tumor formation of $50 \mathrm{~mm}^{3}$. Mice received 5 doses of CBT until the end of MAG-DHA treatment (day 48). B: Tumor growth $\left(\mathrm{mm}^{3}\right)$ as a function of time (day) was measured after subcutaneous injection of $1 \times 10^{6} \mathrm{H} 1299$ cells in control, MAG-DHA, and MAG-DHA+CBT-treated mice. The control mice were sacrificed following tumor formation of $1,000 \mathrm{~mm}^{3}$ on day 35, whereas the mice of MAG-DHA and MAG-DHA+CBT groups were sacrificed on day 54 . Results represent the mean tumor volume $\pm S E M$ ( $n=6$ per group, p<0.05). MAG-DHA, Docosahexaenoic acid monoglyceride; CBT, carboplatin; EGFR, epidermal growth factor receptor; ERK, extracellular signal-regulated kinase.

EPA+DHA ( $2.5 \mathrm{~g} /$ day) of NSCLC patients undergoing platinum chemotherapy resulted in a significant increase in therapy response rate and positive clinical outcomes (12). However, to date no clinical study demonstrated significant effect on tumor size reduction in patient receiving omega-3 supplements in combination with chemotherapeutic agent (10-13).

We therefore, investigated the ability of MAG-DHA and chemotherapy agent CBT to reduce EGFR activation, involved in cell proliferation and invasion. Our current data reveal that MAG-DHA treatment in the presence of CBT markedly decreased EGFR phosphorylation and total EGFR expression levels in both adenocarcinoma cell lines. EGFR is known to activate MAPK superfamily, including the extracellular signal-regulated kinase (ERK). The ERK pathway, activated by mitogenic stimuli such as growth factors, cytokines, and phorbol esters, plays a major role in regulating lung adenocarcinoma cell growth, survival, and differentiation $(14,15-18)$. The decreased expression of EGFR following MAG-DHA+CBT treatment observed herein is concomitant with a decrease in the phosphorylation of ERK and a reduced proliferation level.

\section{Conclusion}

Herein, we demonstrated that in lung A549 and H1299 adenocarcinoma cells combined treatment of CBT plus MAG-DHA exerts a greater inhibitory effect on EGFR phosphorylation and also phosphorylation of ERK, a downstream kinase of EGFR pathway involved in cell proliferation, compared to cells treated with MAG-DHA alone. This inhibition might contribute in reducing tumor growth in A549 and H1299 xenograft mice models. However, further research is still required to determine the mechanisms by which both MAG-DHA and chemotherapeutic agent are mediating their effects. The present findings also warrant further clinical studies to investigate the potential application of MAG-DHA as new and prospective compounds of medicinal interest in the treatment of lung cancer.

\section{Conflicts of Interest}

Samuel Fortin is the owner of SCF Pharma, which has an exclusive worldwide license of the patented uses of MAG-DHA.

\section{Acknowledgements}

The MAG-DHA has been kindly donated by Solutex (Spain).

\section{References}

1 Molina JR, Yang P, Cassivi SD, Schild SE and Adjei AA: Nonsmall cell lung cancer: epidemiology, risk factors, treatment, and survivorship. Mayo Clin Proc 83: 584-594, 2008.

2 D'Eliseo D and Velotti F: Omega-3 fatty acids and cancer cell cytotoxicity: Implications for multi-targeted cancer therapy. J Clin Med 5: E15, 2016.

3 Gleissman H, Johnsen JI and Kogner P: Omega-3 fatty acids in cancer, the protectors of good and the killers of evil? Exp cell Res 316: 1365-1373, 2010.

4 Larsson SC, Kumlin M, Ingelman-Sundberg M and Wolk A: Dietary long-chain n-3 fatty acids for the prevention of cancer: a review of potential mechanisms. The Am J Clin Nutr 79: 935945, 2004.

5 Sun $\mathrm{H}, \mathrm{Hu} \mathrm{Y,} \mathrm{Gu} \mathrm{Z,} \mathrm{Owens} \mathrm{RT,} \mathrm{Chen} \mathrm{YQ} \mathrm{and} \mathrm{Edwards} \mathrm{IJ:}$ Omega-3 fatty acids induce apoptosis in human breast cancer cells and mouse mammary tissue through syndecan-1 inhibition of the MEK-Erk pathway. Carcinogenesis 32(10): 1518-1524, 2011. 
6 Schley PD, Jijon HB, Robinson LE and Field CJ: Mechanisms of omega-3 fatty acid-induced growth inhibition in MDA-MB231 human breast cancer cells. Breast Cancer Res Treat 92: 187195, 2005.

7 Morin C, Rousseau É and Fortin S: Anti-proliferative effects of a new docosapentaenoic acid monoacylglyceride in colorectal carcinoma cells. Prostaglandins Leukot Essent Fatty Acids 89: 203-213, 2013.

8 Morin C, Fortin S, Cantin AM, Sirois M, Sirois C, Rizcallah E and Rousseau É: Anti-cancer effects of a new docosahexaenoic acid monoacylglyceride in lung adenocarcinoma. Recent Pat Anticancer Drug Discov 8: 319-334, 2013.

9 Murphy RA, Mourtzakis M and Mazurak VC: n-3 polyunsaturated fatty acids: the potential role for supplementation in cancer. Curr Opin Clin Nutr Metab Care 15: 246-251, 2012.

10 SL Morland, KJB Martins and VC Mazurak: n-3 polyunsaturated fatty acid supplementation during cancer chemotherapy. Journal of Nutrition \& Intermediary 5: 107-116, 2016.

11 Baracos VE, Mazurak VC and Ma DW: n-3 Polyunsaturated fatty acids throughout the cancer trajectory: influence on disease incidence, progression, response to therapy and cancerassociated cachexia: Nutr Res Rev 17: 177-192, 2004.

12 Murphy RA, Mourtzakis M, Chu QS, Baracos VE, Reiman T and Mazurak VC: Nutritional intervention with fish oil provides a benefit over standard of care for weight and skeletal muscle mass in patients with nonsmall cell lung cancer receiving chemotherapy: Cancer 117: 1775-1782, 2011.

13 Bougnoux P, Hajjaji N, Ferrasson MN, Giraudeau B, Couet C and Le Floch O: Improving outcome of chemotherapy of metastatic breast cancer by docosahexaenoic acid: a phase II trial: Br J Cancer 101: 1978-1985, 2009.

14 Bethune G, Bethune D, Ridgway N and Xu Z: Epidermal growth factor receptor (EGFR) in lung cancer: an overview and update. J Thorac Dis 2: 48-51, 2010.

15 Inamura $\mathrm{K}$, Ninomiya $\mathrm{H}$, Ishikawa $\mathrm{Y}$ and Matsubara $\mathrm{O}$ : Is the epidermal growth factor receptor status in lung cancers reflected in clinicopathologic features? Arch Pathol Lab Med 134: 66-72, 2010 .
16 Ohsaki Y, Tanno S, Fujita Y, Toyoshima E, Fujiuchi S and Nishigaki Y:. Epidermal growth factor receptor expression correlates with poor prognosis in non-small cell lung cancer patients with p53 overexpression. Oncol Rep 7: 603-607, 2000.

17 Veale D, Kerr N, Gibson GJ, Kelly PJ and Harris AL: The relationship of quantitative epidermal growth factor receptor expression in non-small cell lung cancer to long term survival. Br J Cancer 68: 162-165, 1993.

18 Fontanini G, De Laurentiis M, Vignati S, Chinè S, Lucchi M, Silvestri V, Mussi A, De Placido S, Tortora G, Bianco AR, Gullick W, Angeletti CA, Bevilacqua G and Ciardiello F: Evaluation of epidermal growth factor-related growth factors and receptors and of neoangiogenesis in completely resected stage IIIIA non-small-cell lung cancer: amphiregulin and microvessel count are independent prognostic indicators of survival. Clin Cancer Res 4: 241-249, 1998.

19 Fortin S: Polyunsaturated fatty acid monoglycerides, derivatives, and uses thereof. CA2672513, 2008, CA2677670, 2010, US8119690, 2011.

20 Fortin S: Compositions comprising polyunsaturated fatty acid monoglycerides or derivatives thereof and uses thereof. US819690, 2012, US8222295, 2012.

21 van der Meij BS, Langius JA, Spreeuwenberg MD, Slootmaker SM, Paul MA Smit EF and van Leeuwen PA: Oral nutritional supplements containing n-3 polyunsaturated fatty acids affect quality of life and functional status in lung cancer patients during multimodality treatment: an RCT. Eur J Clin Nutr 66: 399-404, 2012.
Received August 21, 2017

Revised September 18, 2017 Accepted September 21, 2017 